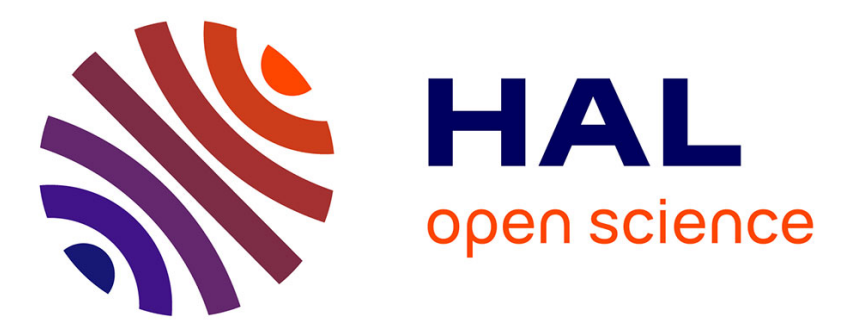

\title{
Le rôle de l'activité de régulation sociale dans le processus d'affirmation de nouvelles parties prenantes: l'exemple des réseaux de promotion de la diversité
} Cathy Krohmer, Christine Naschberger, André Sobczak

\section{- To cite this version:}

Cathy Krohmer, Christine Naschberger, André Sobczak. Le rôle de l'activité de régulation sociale dans le processus d'affirmation de nouvelles parties prenantes: l'exemple des réseaux de promotion de la diversité. Revue management \& avenir, 2010, (33), pp.258-274. 10.3917/mav.033.0258 . hal-00771156

\author{
HAL Id: hal-00771156 \\ https://hal.science/hal-00771156
}

Submitted on 5 Jun 2013

HAL is a multi-disciplinary open access archive for the deposit and dissemination of scientific research documents, whether they are published or not. The documents may come from teaching and research institutions in France or abroad, or from public or private research centers.
L'archive ouverte pluridisciplinaire HAL, est destinée au dépôt et à la diffusion de documents scientifiques de niveau recherche, publiés ou non, émanant des établissements d'enseignement et de recherche français ou étrangers, des laboratoires publics ou privés. 


\title{
Le rôle de l'activité de régulation sociale dans le processus d'affirmation de nouvelles parties prenantes: l'exemple des réseaux de promotion de la diversité
}

\author{
par Cathy Krohmer ${ }^{87}$, Christine Naschberger ${ }^{88}$ \\ et André Sobczak ${ }^{89}$
}

\section{Résumé}

L'objectif de cet article est d'analyser le processus par lequel de nouvelles catégories de parties prenantes peuvent émerger et s'affirmer comme des acteurs suffisamment puissants, légitimes et engagés pour devenir des partenaires des démarches de responsabilité sociale mises en place par les entreprises en général et d'une démarche de promotion de la diversité en particulier. Pour ce faire, l'article mobilise la théorie de la régulation sociale de Jean-Daniel Reynaud qui insiste sur le rôle des acteurs sociaux dans l'émergence et la mise en œuvre des règles régissant la vie dans les organisations, mais qui souligne également qu'à l'occasion de ces régulations, de nouveaux acteurs peuvent émerger et que ceux-ci se constituent et s'affirment par leur contribution à l'activité de régulation.

\section{Abstract}

The aim of this article is to analyze the process through which new categories of stakeholders emerge and constitute themselves as sufficiently powerful, legitimate and committed actors to become partners in companies' strategies in the area of corporate social responsibility and diversity management. To do this, the article mobilizes the theory of social regulation developed by JeanDaniel Reynaud that insists on the role of social actors in the development and enforcement of rules governing organizations and that underlines that these regulations may lead to the emergence of new actors.

Définies comme l'ensemble des individus ou groupes d'individus qui peuvent affecter ou être affectés par la réalisation des objectifs d'une entreprise (Freeman, 1984), les parties prenantes ne se limitent pas aux actionnaires, aux salariés, aux clients et aux fournisseurs, mais incluent de nombreux autres acteurs individuels et collectifs, dont le périmètre exact varie en fonction du contexte juridique, économique, culturel et historique. Ainsi, dans le cadre de sa démarche de responsabilité sociale, l'entreprise doit s'efforcer à dresser régulièrement une

\footnotetext{
87. Cathy Krohmer, Université de Nantes, cathy.krohmer@univ-nantes.fr

88. Christine Naschberger, Audencia Nantes School of Management, Institut pour la Responsabilité Globale dans I'Entreprise, cnaschberger@audencia.com

89. André Sobczak, Audencia Nantes School of Management, Institut pour la Responsabilité Globale dans I'Entreprise, asobczak@ audencia.com
} 


\section{Le rôle de l'activité de régulation sociale dans le processus d'affirmation de nouvelles parties prenantes: l'exemple des réseaux de promotion de la diversité}

cartographie de ces parties prenantes et à identifier leur pouvoir, leur légitimité et l'urgence de leurs intérêts (Mitchell, Agle \& Wood, 1997), voire le degré de leur engagement (Girard \& Sobczak, 2010). Cette définition laisse donc la place à l'émergence de nouvelles catégories ou sous-catégories de parties prenantes dont le rôle ne doit pas être sous-estimé par les managers, alors que ceux-ci pourraient être tentés de concentrer leurs politiques en matière de responsabilité sociale aux acteurs habituels déjà identifiés depuis longtemps.

L'objectif de cet article est d'analyser le processus par lequel de nouvelles catégories de parties prenantes peuvent émerger et s'affirmer comme des acteurs suffisamment puissants, légitimes et engagés pour devenir des partenaires des démarches de responsabilité sociale mises en place par les entreprises. Pour ce faire, nous nous appuyons sur la théorie de la régulation sociale de Jean Daniel Reynaud (1979, 1988, 1997, 1999, 2003 ; Reynaud \& Richebé, 2007) qui insiste sur le rôle des acteurs sociaux dans l'émergence et la mise en œuvre des règles régissant la vie dans les organisations, mais qui souligne également qu'à l'occasion de ces régulations, de nouveaux acteurs peuvent émerger et que ceuxci se constituent et s'affirment par leur contribution à l'activité de régulation.

Pour illustrer ce rôle essentiel de l'activité de régulation sociale dans le processus d'émergence et d'affirmation d'une nouvelle catégorie de parties prenantes, nous avons choisi d'analyser les stratégies et les activités des réseaux qui émergent pour lutter contre les discriminations et pour promouvoir la diversité dans les entreprises. Ces réseaux qui se constituent pour défendre les intérêts de certaines catégories de salariés et de demandeurs d'emploi comme notamment les femmes (Landrieux-Kartochian, 2007), les seniors, les minorités visibles, les homosexuels ou encore les handicapés, s'affirment progressivement comme des nouveaux partenaires pour les entreprises qui, dans le cadre de leur démarche de responsabilité sociale, souhaitent renforcer la diversité de leurs équipes et mettre en place des pratiques de management adaptés à cette diversité.

Le domaine de la lutte contre les discriminations et de la promotion de la diversité qui s'inscrit pleinement dans les démarches de responsabilité sociale semble particulièrement pertinent pour l'analyse du processus d'émergence d'une nouvelle catégorie de parties prenantes. D'une part, les actions mises en place par les entreprises dans ce domaine impliquent la plupart du temps des changements dans les manières de penser et de faire, et donc un engagement des managers, des salariés et des autres parties prenantes, y compris de celles qui émergent, pour identifier et mettre en œuvre des solutions nouvelles (Peretti et Cerdin, 2005) dans le cadre d'un processus d'apprentissage collectif (Berthoin Antal et Sobczak, 2004). D'autre part, ces actions, même si elles correspondent en principe à des initiatives volontaires qui vont au-delà de la loi et des conventions collectives (Commission européenne, 2002), se traduisent souvent par la mise en place de nouvelles régulations sociales pouvant enrichir les normes juridiques 
contraignantes (Sobczak, 2002) et créant l'opportunité pour les parties prenantes impliquées de s'affirmer.

L'article est organisé en trois parties. Dans une première partie, nous présentons les principes de la théorie de la régulation sociale de Jean-Daniel Reynaud, ses principaux apports à une meilleure compréhension des pratiques de management dans les entreprises et ses implications méthodologiques, en montrant ainsi la pertinence de la retenir comme cadre théorique de notre analyse des réseaux de promotion de la diversité. Dans une deuxième partie, nous soulignons la diversité des parties prenantes impliquées dans la promotion de la diversité et l'élaboration de nouvelles règles dans ce domaine, en distinguant entre les acteurs traditionnels de la régulation sociale, à savoir les pouvoirs publics et les partenaires sociaux, des nouvelles parties prenantes que constituent les réseaux de promotion de la diversité. Enfin, dans une troisième partie, nous présentons les résultats d'une étude exploratoire sur les stratégies et les activités de différents réseaux de promotion de la diversité, en mettant en évidence leur contribution à l'émergence de nouvelles règles sociales et l'importance que cette activité représente pour leur affirmation en tant que nouvelle partie prenante dont les intérêts doivent être intégrés par les entreprises.

\section{Mobiliser la théorie de la régulation sociale pour analyser l'émergence de nouvelles parties prenantes}

L'apport de la théorie de la régulation sociale de Reynaud est de plus en plus reconnu dans les recherches en sciences de gestion, en particulier dans le domaine du management stratégique (Brechet, 2008), de la gestion des ressources humaines (Havard \& Krohmer, 2008) ou encore du management de la responsabilité sociale des entreprises (Sobczak, 2010). Les pratiques de management, y compris celles dans le domaine de la diversité, peuvent être analysées comme le résultat de régulations au sens de Reynaud, enrichissant ainsi leur compréhension.

Tout en reconnaissant la diversité des règles qui régissent une organisation, la théorie de la régulation sociale remet en cause la distinction entre les règles formelles - dont les règles juridiques sont une mais pas la seule illustration - et les règles informelles (Reynaud, 1988). Elle propose d'y substituer une nouvelle classification, en distinguant entre la régulation autonome et la régulation de contrôle. La première correspond aux règles que le groupe social considéré se donne lui-même. Le terme autonome a été retenu par Reynaud pour souligner la capacité des acteurs à affirmer leur autonomie à l'égard des autres acteurs qui tentent de leur imposer des règles de l'extérieur. Qualifiées de régulation de contrôle, ces règles émanant de l'extérieur du groupe visent à orienter et prescrire les comportements de celui-ci et à contrôler les zones d'autonomie de ses membres. 


\section{Le rôle de l'activité de régulation sociale dans le processus d'affirmation de nouvelles parties prenantes: l'exemple des réseaux de promotion de la diversité}

Cette nouvelle distinction présente le mérite de mettre en évidence l'importance de l'activité de régulation pour les acteurs sociaux. Si la régulation est définie comme la capacité de prendre des initiatives et d'élaborer des règles (Reynaud, 1988), l'activité de régulation peut être analysée comme une manière pour les acteurs sociaux de se constituer et de s'affirmer. En créant des règles, les acteurs sociaux élaborent une stratégie collective et s'insèrent dans un jeu social. Ils définissent leur étendue et leurs limites, prononcent des exclusions qui font leur spécificité, précisent leurs structures internes et disent à qui ils s'allient et à qui ils s'opposent (Reynaud, 1988). L'intérêt accordé par la théorie de la régulation sociale aux acteurs dans les processus de régulation explique aussi l'accent qu'elle met sur les relations de pouvoir, en particulier entre les salariés et les représentants du management (Reynaud, 1979 et 1988).

La théorie de la régulation sociale souligne par ailleurs le caractère très dynamique des différentes régulations régissant une organisation (Reynaud, 1991). Aussi bien la régulation autonome que la régulation de contrôle présentent une instabilité liée à celle des acteurs sociaux eux-mêmes. Selon Reynaud (1991), les régulations « sont sans cesse produites, corrigées, affaiblies ou renforcées par les acteurs sociaux. II n'y a pas de règles stables mais seulement des processus de régulation ॥. Cette approche semble particulièrement adaptée à l'analyse des pratiques de responsabilité sociale mises en œuvre dans les entreprises qui doivent évoluer en permanence pour tenir compte des évolutions des intérêts et attentes des parties prenantes. La théorie de la régulation sociale met notamment en lumière le rôle des conflits dans l'évolution de la régulation. Selon (Reynaud, 1979), la régulation ne s'oppose pas au conflit mais en est la solution provisoire acceptée par les acteurs sociaux. Dès lors, il considère que « l'étude du conflit est indispensable pour comprendre la portée et la solidité de cette solution » (Reynaud, 1979). L'étude du conflit entre les acteurs sociaux permet de mieux comprendre les limites et les faiblesses de la régulation et de faire des hypothèses sur l'évolution possible de cette régulation. Cette perspective peut, là encore, enrichir l'analyse de pratiques en matière de responsabilité sociale des entreprises dans la mesure où celle-ci peut souvent impliquer des conflits d'intérêts entre les différentes parties prenantes.

La théorie de la régulation sociale suggère enfin que, si la régulation autonome et la régulation de contrôle peuvent coexister en parallèle et entrer alors en concurrence, elles peuvent aussi donner lieu à la négociation implicite ou explicite d'une régulation conjointe, acceptée par les acteurs sociaux en jeu (Reynaud, 2003). La négociation collective entre les partenaires sociaux pour définir des règles régissant les relations de travail est un exemple parmi d'autres d'une régulation conjointe (Reynaud, 2005).

Cette présentation très succincte des principes de la théorie de la régulation sociale illustre sa possible contribution à une meilleure compréhension des pratiques 
en matière de management de la diversité et de l'émergence des nouveaux acteurs dans ce domaine. Les pratiques visant à promouvoir la diversité peuvent être analysées comme des formes de régulation au sens de Reynaud. Selon la perspective, elles peuvent être considérées comme des régulations autonomes, des régulations de contrôle ou des régulations conjointes. Ainsi, les accords sur la diversité négociés entre les entreprises et les organisations syndicales sont d'abord une forme de régulation conjointe. Mais une fois qu'ils sont conclus, ces accords doivent se transformer en pratiques de management qui s'imposent aux managers et salariés en tant que régulation de contrôle. D'autres pratiques de promotion de la diversité peuvent directement émerger au sein d'un groupe d'acteurs au sein de l'entreprise, constituant alors une régulation autonome. C'est le cas en particulier des actions menées par certains réseaux de promotion de la diversité qui peuvent être internes à une entreprise et qui peuvent s'appuyer sur cette régulation pour se constituer en tant que partie prenante légitime dans ce domaine.

\section{Comprendre le rôle des parties prenantes dans la promotion de la diversité}

Dans le cadre de leurs démarches de responsabilité sociale, de nombreuses entreprises mettent en place des actions visant à lutter contre les discriminations ou à promouvoir la diversité. Ces actions ont pour objectif de « faire évoluer les représentations pour éliminer tout comportement discriminatoire dans l'entreprise et instaurer une culture de tolérance, qui permette l'inclusion de chacun avec ses apports et ses différences » (Bender, 2007). II s'agit donc de « planifier et mettre en œuvre des systèmes et des pratiques organisationnelles pour manager les Hommes de sorte que les avantages potentiels de la diversité soient maximisés tandis que ses inconvénients potentiels sont réduits au minimum " (Cox, 1993 - notre traduction). Le management de la diversité met ainsi l'accent sur la reconnaissance et la valorisation des différences individuelles et, à ce titre, considère chaque individu comme une valeur ajoutée pour la performance de l'entreprise.

L'importance des enjeux du management de la diversité pour l'ensemble de la société explique que de très nombreuses parties prenantes se mobilisent. Après avoir analysé le rôle joué dans ce domaine par les pouvoirs publics et les partenaires sociaux (2.1.), nous nous concentrons sur les réseaux de promotion de la diversité et leur contribution spécifique par rapport aux autres acteurs (2.2.). 

dans le processus d'affirmation de nouvelles parties prenantes : l'exemple des réseaux de promotion de la diversité

\subsection{Le rôle des parties prenantes traditionnelles : les pouvoirs publics et des partenaires sociaux}

Dans la mesure où elles rentrent dans le champ de la régulation sociale, la lutte contre les discriminations et la promotion de la diversité dans le monde du travail ont fait l'objet de multiples actions de la part des pouvoirs publics et ses partenaires sociaux, les trois acteurs traditionnels de cette régulation.

Le droit du travail français imposé par les pouvoirs publics contient depuis longtemps des règles sur la lutte contre les discriminations, aussi bien au moment de l'embauche que pendant toute la durée de l'exécution du contrat de travail. Le champ d'application de ces règles s'est progressivement étendu, notamment sous l'influence du droit social européen, pour multiplier les critères de discrimination interdits et inclure tous les aspects de la relation de travail. Pour donner plus de poids à la lutte contre les discriminations, le législateur a, à plusieurs reprises, renforcé les sanctions pénales en cas de violation de ces règles du droit du travail. De même, le législateur, conscient des difficultés pour les victimes d'apporter la preuve de la discrimination, a modifié les règles de preuve dans ce domaine. Dès lors que le salarié présente des éléments de faits laissant supposer l'existence d'une discrimination, c'est à l'employeur d'apporter la preuve de l'absence de discrimination, en faisant état d'éléments objectifs justifiant les différences de traitement. Le législateur a ainsi décidé de renverser la charge et le risque de la preuve qui incombent en principe au demandeur. Allant encore plus loin, le droit du travail admet que les procédures soient introduites non pas par la victime elle-même, mais par des organisations syndicales ou des associations engagées dans la promotion de la diversité.

Conformément aux obligations découlant du droit européen, la France a par ailleurs créé en 2004 une autorité administrative indépendante chargée de lutter contre les discriminations et de promouvoir l'égalité des chances. La Haute Autorité de Lutte contre les Discriminations et pour l'Egalité des chances (HALDE) peut être saisie par toute personne s'estimant victime d'une discrimination. Elle dispose d'un pouvoir d'enquête dans les entreprises et peut jouer un rôle de médiation hors des instances judiciaires ou alors intervenir dans un procès en appui de la victime, notamment en l'aidant à rassembler des éléments de preuve.

Si le cadre juridique en matière de lutte contre les discriminations a été considérablement renforcé, notamment depuis 2001, force est de constater que de nombreuses différences de traitement subsistent. De multiples initiatives de testing ont montré que la chance d'être invité à un entretien de recrutement varie fortement en fonction du sexe du candidat, de son origine, vraie ou supposée, voire de son adresse. Certes, on voit une multiplication des procédures à la fois devant les juridictions et devant la HALDE, mais la seule action des pouvoirs publics ne peut suffire pour garantir l'égalité de traitement, voire une vraie diversité dans les entreprises. Cela est d'autant plus vrai que les pouvoirs publics français 
restent pour l'instant opposés à la mise en place de mesures de discrimination positive pourtant autorisées par le droit européen et pratiquées dans d'autres Etats membres, voire même à l'établissement de statistiques ethniques.

II est dès lors important que les partenaires sociaux se soient également saisis depuis quelques années du sujet. Au-delà de l'accord national interprofessionnel (ANI) du 11 octobre 2006 sur la diversité dans l'entreprise (IMS-Entreprendre pour la Cité, 2006 ; Cornet et Warland, 2008), de nombreux accords d'entreprise ont été conclus sur l'égalité professionnelle, l'intégration des personnes en situation de handicap, voire sur la diversité en général. On peut par exemple citer les accords signés chez PSA Peugeot Citroën ${ }^{90}$ ou chez HSBC ${ }^{91}$. Certaines entreprises ont décidé de faire de la promotion de la diversité l'un des premiers sujets d'un dialogue social transnational. Total a signé un accord européen sur la diversité avec son comité d'entreprise européen ${ }^{92}$, Danone un accord mondial sur la diversité avec l'UITA ${ }^{93}$. Cet accord rappelle que la diversité est une richesse et se décline en chapitres relatifs aux différents domaines de la Gestion des Ressources Humaines. De même, PSA Peugeot Citroën a intégré une partie sur l'interdiction des discriminations et la promotion de l'égalité des chances dans son accord mondial sur la responsabilité sociale.

Au-delà des régulations imposées par les pouvoirs publics ou négociées entre les partenaires sociaux, les entreprises mettent en place de nombreuses actions volontaires pour lutter contre les discriminations ou pour promouvoir l'égalité des chances et la diversité qui complètent les règles du droit du travail et les accords collectifs dans ce domaine. De nombreuses normes et standards élaborés au niveau international que les entreprises peuvent volontairement décider de respecter en matière de responsabilité sociale font référence à cette thématique. Le Global Compact, une initiative des Nations Unies invitant les entreprises du monde entier à s'engager volontairement à respecter et à promouvoir dix principes dans le domaine des droits de l'homme, des droits sociaux, de la protection de l'environnement et de la lutte contre la corruption, contient ainsi un principe relatif à l'interdiction de toutes les discriminations, en se référant aux normes de l'Organisation Internationale du Travail sur ce sujet. Les principes directeurs de l'Organisation de Coopération et de Développement Economiques à destination des entreprises multinationales qui s'adressent autant aux Etats qu'aux entreprises contiennent une norme relative à la lutte contre les discriminations. Cela est aussi vrai pour le label privé SA 8000 qui permet de certifier des sites de production respectant un ensemble de normes relatives aux relations de travail.

\footnotetext{
90. Accord sur le développement de l'emploi féminin et l'égalité professionnelle entre les femmes et les hommes ; accord sur la diversité et la cohésion sociale dans l'entreprise de septembre 2004, renouvelé en octobre 2008.

91. Accord triennal sur l'égalité professionnelle entre les hommes et les femmes du 28 février 2003 ; accord triennal sur l'insertion des handicapés dans l'entreprise du 3 juin 2004 ; accords salariaux depuis 2004 incluant des mesures notamment sur la gestion des augmentations salariales après des absences de longue durée (ex : congé maternité) et mise en place d'enveloppes financières (égalité professionnelle, retour de congé maternité, handicapés, salariés de plus de 50 ans) ; accord global sur la diversité de fin décembre 2007.

92. Accord européen sur l'égalité des chances du 21 novembre 2005.

93. Convention mondiale sur la diversité du 8 juin 2007.
} 

promotion de la diversité

Le thème de la lutte contre les discriminations et de la promotion de la diversité occupe aussi une place très importante dans les normes de responsabilité sociale adoptées au niveau des entreprises. Selon une étude récente pour la Fondation européenne de Dublin, plus de 9 codes de conduite sur 10 adoptés unilatéralement par les directions d'entreprise en matière de responsabilité sociale font référence à ce sujet, et il en va de même pour les accords-cadre internationaux négociés entre des entreprises et les fédérations syndicales internationales (Schoemann \& al., 2008).

\subsection{Le rôle d'une nouvelle catégorie de parties prenantes : les réseaux de promotion de la diversité}

Les théories des réseaux sociaux montrent que les individus n'agissent pas seuls et de manière isolée, mais sont insérés à l'intérieur de réseaux de relations qui peuvent soit limiter, soit faciliter leurs actions (Baret, Huault et Pick, 2006). Plusieurs recherches mettent ainsi en évidence l'importance du rôle des réseaux dans les processus de recrutement et l'évolution de la carrière. II est donc important de faire partie du ou des "bons réseaux " (Chollet, 2006) qui augmentent les opportunités d'emploi et de carrière pour leurs membres et leur permettent d'accéder à des rémunérations plus élevées.

Or, plusieurs recherches mettent en évidence le fait que certaines catégories sont exclues des réseaux performants permettant d'accéder aux positions hiérarchiques et aux rémunérations les plus élevées. Ainsi, pour Laufer (2005) l'existence des discriminations dans le monde du travail entre hommes et femmes, s'explique en partie par la faible insertion de ces dernières dans les réseaux. Selon LandrieuxKartochian (2007), les femmes ont plus de difficultés que les hommes à accéder à des réseaux, à les animer et à s'en servir comme accélérateur de carrière. De même, Brass (1985) montre que les femmes ne sont pas bien intégrées dans les réseaux d'hommes, réseaux qui justement peuvent permettre d'accéder à des positions plus élevées. Le constat est similaire pour les minorités visibles. Dans une étude sur les salaires négociés dans les entreprises américaines de haute technologie entre 1985 et 1995, Seidel et al. (2000) mettent en évidence que le fait d'avoir dans son réseau des membres de l'entreprise que l'individu souhaite rejoindre, augmente le niveau du salaire négocié à l'embauche. Or, les réseaux des minorités visibles ne comportent souvent pas ces personnes.

D'autres recherches montrent au contraire que les « bons réseaux » peuvent limiter les risques de discrimination. Ainsi, Marchal et Rieucau (2009) ont évalué l'incidence du mode de recrutement sur le risque de discrimination, en comparant les chances des candidats ayant répondu à une offre d'emploi à celles des candidats introduits par un réseau de relations. Ils révèlent que des candidats qui sont potentiellement victimes de discriminations liées à leur âge, à leur diplôme, voire à leur nationalité, ont plus de chances d'accéder à un emploi s'ils s'appuient 
sur des relations plutôt que de répondre à des annonces. L'appui du réseau de relations permet plus facilement de passer outre les critères pouvant donner lieu à des discriminations pour porter le débat directement sur les compétences inobservables comme la motivation, le savoir-faire, les capacités relationnelles ou la conscience professionnelle.

Ibarra (1993) introduit deux distinctions parmi les réseaux sociaux. La première distinction oppose les réseaux prescrits ou formels aux réseaux émergents ou informels. Un réseau prescrit est composé d'un ensemble de relations formellement spécifiées entre des supérieurs et des subordonnés et entre des groupes ayant des fonctions différentes qui doivent interagir pour accomplir des tâches définies par l'organisation. Par contraste, les réseaux informels relèvent de combinaisons plus discrètes, les contenus des relations pouvant être liés au travail, aux relations sociales ou aux deux à la fois. La deuxième distinction oppose les réseaux aux liens instrumentaux ou faibles aux réseaux expressifs supportés par des liens d'amitiés ou sociaux qui correspondent à des liens forts. Les réseaux instrumentaux visent à améliorer la performance au travail et incluent l'échange de ressources liées au travail, comme des informations, de l'expertise, des conseils professionnels, des accès politiques ou des ressources matériels. Au contraire, les réseaux expressifs impliquent l'échange de supports amicaux et sociaux et sont caractérisés par un plus haut niveau de proximité et de confiance que ceux qui sont exclusivement instrumentaux. S'appuyant sur une étude réalisée auprès de 300 cadres qui venaient de changer d'emploi, Granovetter (1973), montre l'efficacité des liens faibles et instrumentaux par rapport aux liens forts dans la recherche d'emploi, en soulignant que les salariés ont plus souvent connaissance des opportunités d'emploi grâce à des liens faibles que grâce à des liens forts.

L'importance du rôle des réseaux a conduit au développement d'une nouvelle catégorie de parties prenantes, les réseaux de promotion de la diversité. Ces réseaux complètent les actions mises en place par les pouvoirs publics, les organisations syndicales et les entreprises pour lutter contre les discriminations et promouvoir la diversité. Ils se caractérisent par une grande variété de leurs membres et de leurs objectifs.

Une première distinction peut être faite entre les réseaux catégoriels et ceux ayant un objectif plus général de promotion de la diversité. La plupart des réseaux sont catégoriels et réunissent des individus ayant en commun une caractéristique qui peut être cause de discriminations lors d'une procédure de recrutement ou au cours de la carrière. II y a ainsi des réseaux regroupant des femmes (par exemple : Business au Féminin, European Profesional Women Network), des minorités visibles (par exemple : Africagora, Association pour Favoriser l'Intégration Professionnelle) ou des homosexuels (par exemple : Autre Cercle, Comin-G). Ces réseaux se concentrent sur les problèmes spécifiques 

promotion de la diversité

rencontrés par chacune de ces catégories. D'autres réseaux ont une vocation plus générale de lutte contre les discriminations et de promotion de la diversité et regroupent des membres représentant différentes catégories (par exemple : IMS Entreprendre).

Une autre distinction peut être établie entre les réseaux organisés au niveau d'une entreprise et ceux qui regroupent des demandeurs d'emploi ou des salariés de plusieurs entreprises. Si les réseaux de promotion de la diversité se sont d'abord développés en dehors des entreprises par des victimes de discriminations dans le monde du travail, on constate depuis quelques années l'émergence de réseaux internes à certaines entreprises, souvent avec un soutien important de la part de la direction des ressources humaines, voire de la direction générale, conscientes des enjeux du management de la diversité. On peut notamment mentionner les réseaux «Elles » et « Employee Alliance for Gay, Lesbian, Bisexual and Transgender Empowerment (Eagle) » au sein d'IBM ou le réseau « Accent sur Elles » chez Accenture.

Enfin, on peut distinguer les réseaux regroupant des victimes de discriminations au moment de l'accès à l'emploi ou au cours de leur carrière, des réseaux rassemblant des dirigeants et managers qui souhaitent améliorer les pratiques au sein de leurs entreprises (par exemple : IMS Entreprendre pour la Cité, Association Française des Managers de la Diversité). Si les premiers se fixent avant tout pour objectif de soutenir leurs membres dans la défense de leurs intérêts en tant que demandeurs d'emploi ou salariés, notamment en donnant des conseils ou en organisant des formations, les seconds cherchent à favoriser l'échange d'expériences et la sensibilisation des équipes de leurs entreprises. Pour autant, les deux types de réseaux peuvent parfois coopérer, par exemple sur l'organisation de forums de recrutement réservés à certaines catégories de demandeurs d'emplois, souvent victimes de discriminations.

\section{Mesurer l'importance de l'activité de régulation sociale pour les réseaux de promotion de la diversité}

Pour tester la pertinence d'une analyse de l'activité des réseaux de promotion de la diversité en nous fondant sur la théorie de la régulation sociale, nous avons conduit une enquête exploratoire reposant sur deux méthodologies complémentaires.

D'une part, entre février et septembre 2007, nous avons mené une série de 11 entretiens semi-directifs avec les représentants de différents types de réseaux de promotion de la diversité sur les objectifs et les actions des réseaux ainsi que sur leurs résultats. Ces entretiens devaient nous permettre de mieux comprendre les actions de ces réseaux et leur implication en matière de pratique de diversité. 
Les guides d'entretien portaient sur la présentation du réseau et de ses objectifs, sur les actions mises en œuvre ainsi que sur leur impact sur le management de la diversité dans les entreprises. Les réseaux que nous avons inclus dans cette étude exploratoire ont été choisis en raison de leur implication dans les actions facilitant l'accès aux institutions d'enseignement supérieur, notamment les Grandes Ecoles, que cela soit en formation initiale pour faciliter l'accès à l'emploi ou en formation continue pour progresser tout au long de la carrière. L'échantillon comportait des réseaux représentant une grande variété de catégories de salariés ou de demandeurs d'emploi victimes de discriminations (voire tableau 1).

Tableau 1: Réseaux de promotion de la diversité inclus dans l'enquête exploratoire

\begin{tabular}{|c|c|}
\hline Nom du réseau & Description \\
\hline AFIP & $\begin{array}{l}\text { Association pour Favoriser l'Intégration Professionnelle, favorise l'accès } \\
\text { à l'emploi des jeunes diplômés issus des minorités visibles }\end{array}$ \\
\hline Africagora & $\begin{array}{l}\text { Africagora est un club et une association composés d'entrepreneurs, de } \\
\text { cadres et d'élus originaires d'Afrique, des Caraïbes et du Pacifique, et } \\
œ u v r a n t \text { pour l'intégration économique, l'insertion professionnelle et la } \\
\text { promotion sociale des minorités ethniques. }\end{array}$ \\
\hline Comin-G & $\begin{array}{l}\text { COMIN-G est l'association des personnels lesbiens, gays, bi et trans. } \\
\text { (LGBT) du Ministère de l'Economie, des Finances et de l'Industrie }\end{array}$ \\
\hline Companieros & $\begin{array}{l}\text { Companieros est un acteur éducatif innovant, qui propose aux grandes } \\
\text { écoles et universités, avec le soutien d'entreprises partenaires, des } \\
\text { programmes pédagogiques à valeur ajoutée humaine, sociale et } \\
\text { sociétale }\end{array}$ \\
\hline DiverCité & Réseau de gays et de lesbiennes d'une institution académique \\
\hline $\begin{array}{l}\text { IMS Entreprendre } \\
\text { pour la Cité }\end{array}$ & $\begin{array}{l}\text { IMS-Entreprendre pour la Cité fédère un réseau de } 200 \text { entreprises. Sa } \\
\text { vocation est de les aider à intégrer, dans leur politique de Responsabilité } \\
\text { Sociale, des démarches d'Engagement Sociétal innovantes, répondant } \\
\text { à la fois à leurs enjeux de développement et aux attentes de la Société. }\end{array}$ \\
\hline In \& Out & Réseau de gays et lesbiennes d'une institution académique \\
\hline $\begin{array}{c}\text { European } \\
\text { Professional Women } \\
\text { Network (EPWN) }\end{array}$ & Réseau de femmes cadres supérieurs et entrepreneurs en Europe \\
\hline $\begin{array}{l}\text { Grandes Ecoles au } \\
\text { Féminin }\end{array}$ & $\begin{array}{l}\text { Réseau qui réunit des représentants des associations d'anciens élèves } \\
\text { de } 9 \text { grandes écoles }\end{array}$ \\
\hline Réseau Elles & $\begin{array}{l}\text { Réseau intra-entreprise qui regroupe certaines femmes d'une } \\
\text { entreprise }\end{array}$ \\
\hline Réseaudencia & Réseau des Anciens d'une institution académique \\
\hline
\end{tabular}

D'autre part, nous nous appuyons sur une observation participante (Chanlat, 2005 ; Delamont, 2004 ; Groleau, 2003) dans un groupe visant à promouvoir la diversité et réunissant des membres du Centre des Jeunes Dirigeants de Nantes 


\section{Le rôle de l'activité de régulation sociale dans le processus d'affirmation de nouvelles parties prenantes : l'exemple des réseaux de promotion de la diversité}

(CJD) de l'Union Régionale de la CDFT et d'une institution académique. Cette observation participante a été réalisée par les auteurs depuis septembre 2006, date de création du groupe. A l'origine, ce groupe était un groupe d'échange sur la question de la diversité. Peu à peu, une représentation commune de la façon dont doit être mise en œuvre une démarche diversité à émerger. Depuis janvier 2009, le groupe travaille sur l'évaluation et l'amélioration des pratiques de management de la diversité au sein de six entreprises dont le dirigeant est membre du CJD.

De ces enquêtes se dégagent plusieurs résultats. Tout d'abord, les réseaux de promotion de la diversité, comme indiqué par la littérature, permettent aux individus d'accéder à un certain nombre de ressources. Ces réseaux sont d'abord des lieux d'échanges et de sensibilisation. Ainsi, l'European Professional Women Network $(E P W N)$ organise de manière régulière des groupes d'échange et conférences relatives à la carrière au féminin. Ces réseaux offrent aussi à leurs membres des formations spécifiques renforçant leurs compétences et leur attractivité pour les entreprises. De même, l'Association pour Favoriser l'Intégration Professionnelle (AFIP) propose aux jeunes diplômés issus des minorités visibles de se former aux entretiens d'embauche. L'EPWN organise des formations spécifiques pour les femmes sur des thèmes comme la "gestion de carrière au féminin " ou le "leadership au féminin ». Réunissant des entreprises engagées dans des démarches de diversité, IMS Entreprendre pour la Cité dispense pour sa part des formations pour les managers pour leur permettre d'identifier les préjugés et de mieux connaître le cadre règlementaire dans le domaine des discriminations. Les réseaux proposent aussi des actions de mentoring et de coaching. L'AFIP propose ainsi un programme de parrainage mettant en contact des diplômés issus des minorités visibles avec des parrains travaillant dans les entreprises qui s'engagent à « ouvrir leurs carnets d'adresse ». Cet outil est aussi utilisé par Africagora. De même, l'EPWN multiplie les opérations de mentoring.

Nos enquêtes ont cependant révélé que les réseaux de promotion de la diversité contribuent par ailleurs activement à la construction de nouvelles règles dans le domaine du management de la diversité. Par exemple, IMS Entreprendre pour la Cité a été à l'origine de la Charte de la Diversité que les entreprises peuvent adopter pour formaliser leur engagement en faveur de la diversité. Ce réseau a également mis en place un certain nombre de groupes de travail sur les bonnes pratiques développées et les difficultés rencontrées. II s'agit dans ce cas de développer des lieux d'échanges où les acteurs - en l'occurrence des managers des ressources humaines - confrontent leurs pratiques.

Les réseaux de promotion de la diversité peuvent contribuer à l'émergence d'une régulation autonome. C'est ainsi que l'EPWN se fixe pour objectif de mieux accompagner les femmes dans la gestion quotidienne de leurs obligations familiales, en travaillant par exemple sur la garde d'enfants. Les réseaux peuvent 
aussi intervenir dans l'élaboration et le développement des nouvelles pratiques de management de la diversité, devant alors acteurs d'une régulation conjointe. Ainsi, le réseau Elles d'IBM travaille avec la direction des ressources humaines de cette entreprise pour mettre en place des emplois familiaux par IBM visant à faciliter la vie quotidienne dans le domaine de l'enfance, des parents et des personnes handicapées, aidant par exemple les salariés à choisir le mode de garde adéquat ou à mener certaines démarches administratives et juridiques.

Si les entretiens avec les représentants des réseaux de promotion de la diversité montrent que ces réseaux deviennent des acteurs de la régulation, l'observation participante menée dans un réseau émergeant permet de mieux analyser le rôle que joue cette activité de régulation dans son processus de constitution et d'affirmation. Le réseau réunissant des membres du CJD, de la CFDT et d'une institution académique s'est progressivement constitué une représentation commune du management de la diversité. Dès le début, le groupe s'est fixé comme objectif la formation et la diffusion de leur idée auprès d'un large public. C'est ainsi que les membres du réseau ont dispensé des formations aux étudiants de l'institution académique. La réalisation d'un support commun a été l'occasion de formaliser un certain nombre de représentations. Par exemple, les raisons de travailler ensemble ont été explicitées de la manière suivante : " Ensemble, nous souhaitons réfléchir à la façon de mieux intégrer la diversité dans la vie économique, sensibiliser les futurs managers sur cette nécessité, faire partager nos conclusions et témoigner d'une coopération réussie entre dirigeants, syndicalistes et enseignants-chercheurs » (Observation participante).

Ensuite, lors de réunions bimensuelles, les membres du réseau ont analysé d'une manière plus approfondie les freins et les leviers du management de la diversité dans les entreprises, en distinguant selon les problèmes spécifiques à différentes catégories de demandeurs d'emploi ou de salariés comme les femmes, les seniors, les personnes en situation de handicap ou les minorités visibles (voir Naschberger, 2008, pour un exemple de ces réflexions). La création d'une plaquette présentant le groupe en juin 2007 a été l'occasion de développer un slogan - « Tous acteurs pour la diversité dans l'entreprise » - et d'affirmer une vision commune de la manière dont les entreprises devraient, selon le réseau, concevoir et mettre en œuvre leurs démarches de management de la diversité. Cette vision accorde une place primordiale au rôle du dialogue social entre managers et représentants des salariés dans ces démarches. En juin 2008, les membres du réseau ont décidé de lancer des expérimentations, en proposant à des entreprises de se faire accompagner dans leurs démarches de diversité par des étudiants de l'institution académique encadrés par l'ensemble du réseau. A travers ces différents éléments, les acteurs se sont donc découvert un sens commun et des règles communes, conformément aux principes de la théorie de la régulation sociale (Reynaud, 1997). 


\section{Le rôle de l'activité de régulation sociale dans le processus d'affirmation de nouvelles parties prenantes : l'exemple des réseaux de promotion de la diversité}

Plus largement, les deux études exploratoires confirment l'intérêt de l'analyse des réseaux de promotion de la diversité, en s'appuyant sur la théorie de la régulation sociale. La plupart des recherches s'appuient sur les théories des réseaux sociaux pour insister avant tout sur le fait que les victimes des discriminations sont souvent exclues de certaines ressources et donc pour analyser les réseaux de promotion de la diversité comme un moyen de combler cette lacune. Cependant, force est de constater que les réseaux de promotion de la diversité ne disposent souvent pas des éléments identifiés par la recherche comme garantissant la performance d'un réseau en termes d'accès à un emploi ou une carrière de haut niveau. Cela est vrai notamment pour certains réseaux catégoriels qui regroupent des individus victimes de discriminations et qui manquent donc souvent de liens avec les personnes ayant un pouvoir de décision au sein des entreprises. Bras (1985) montre ainsi que les difficultés que les femmes rencontrent dans leur carrière peuvent en partie s'expliquer par le fait que peu d'entre elles appartiennent à des réseaux mixtes comportant des hommes qui ont plus de probabilités de se trouver à des postes de décision. Cette analyse peut d'ailleurs conduire les réseaux de promotion de la diversité à s'ouvrir ou à créer des partenariats avec des entreprises ou des réseaux de managers.

La théorie de la régulation sociale permet de développer un regard complémentaire sur les réseaux de promotion de la diversité, en mettant en évidence leur rôle dans la création ou la modification des règles du jeu dans ce domaine. Au-delà de leur capacité à aider les victimes de discriminations à accéder à des ressources, ces réseaux cherchent à influencer les pratiques de management des entreprises qui peuvent s'analyser comme des règles dans la mesure où elles visent à modifier le comportement des salariés. Cette activité de régulation sociale leur permet de se constituer en insérant les réseaux dans le jeu social et en traduisant leur stratégie collective (Reynaud, 1999).

La plupartdutemps, les pratiques de managementdela diversité peuvents'analyser comme des règles de contrôle imposées par la direction des ressources humaines ou alors par le droit du travail. De leur côté, certains réseaux de promotion de la diversité développent des règles autonomes qui visent à modifier les pratiques des entreprises, mais aussi à s'appuyer sur cette activité de régulation pour se constituer. Parfois, ces règles autonomes portées par ces réseaux sont intégrées et prises en compte par les dirigeants d'entreprise, traduisant alors une forme de régulation conjointe. Dans la mesure où ils contribuent à modifier les règles en vigueur dans les entreprises, les réseaux de promotion de la diversité sont donc des acteurs à part entière du management de la diversité.

\section{Conclusion}

Le développement des pratiques de management de la diversité s'accompagne de l'émergence et de l'affirmation d'une nouvelle catégorie de parties prenantes : 
les réseaux de promotion de la diversité dont les activités contribuent à influencer ces pratiques. Pour mieux comprendre le rôle joué par cette nouvelle partie prenante, nous avons mené une étude exploratoire fondée sur deux enquêtes qualitatives, et analysé les données ainsi récoltées en nous appuyant sur la théorie de la régulation sociale de Jean-Daniel Reynaud qui permet d'appréhender les règles et leur développement dans les organisations. Cette étude exploratoire confirme l'intérêt de recourir à ce cadre théorique de manière complémentaire à celui habituellement mobilisé qui se concentre sur le rôle que les réseaux de promotion de la diversité jouent dans l'accès des victimes de discriminations à certaines ressources. Elle montre que certains réseaux contribuent fortement à l'élaboration et la modification de la régulation sociale et s'appuient sur cette activité pour se constituer ou s'affirmer. Pour poursuivre cette analyse, l'objectif est de conduire dans une prochaine étape une étude approfondie de l'impact des activités d'un ou de plusieurs réseaux de promotion de la diversité sur l'évolution des pratiques de management d'une entreprise donnée.

Néanmoins, il est déjà possible d'identifier certaines implications de cette étude exploratoire pour les managers en charge des démarches de diversité dans leurs entreprises comme pour ceux qui animent un réseau de promotion de la diversité ou plus généralement une nouvelle catégorie de parties prenantes. Pour les managers, il semble important de reconnaître le rôle des réseaux de promotion de la diversité dans l'émergence et la modification des règles dans ce domaine et donc la nécessité d'établir avec eux des relations de confiance. Notre étude peut les conduire à élargir leurs coopérations avec ces réseaux au-delà des actions de recrutement afin de chercher à créer ensemble un processus d'apprentissage commun permettant de définir et de mettre en œuvre de nouvelles pratiques pour lutter contre les discriminations et favoriser l'égalité des chances. La prise de conscience du rôle des réseaux de promotion de la diversité dans le processus de régulation sociale est évidemment aussi utile pour les animateurs de ces réseaux. Ceux-ci ne devraient pas négliger l'importance que cette activité présente pour leur affirmation en tant que partie prenante légitime, en complément de leurs actions visant à aider les victimes des discriminations à accéder à certaines ressources. Comme pour toute nouvelle catégorie de parties prenantes, l'énergie investie dans les processus de régulation sociale peut parfois sembler éloignée des priorités concrètes à traiter à court terme, mais constitue souvent une stratégie nécessaire pour permettre de maintenir et de renforcer l'impact à moyen terme sur les pratiques des entreprises.

\section{Bibliographie}

Baret C., Huault I. et Pick T. (2006). « Management et réseaux sociaux. Jeux d'ombres et de lumières sur les organisations », Revue Française de Gestion, No 163, 93-106.

Bender A.F. (2007). "L'approche diversité dans les pays anglo-saxons », in I. Barth et C. Falcoz, Le management de la diversité, Enjeux, fondements et pratiques, L'Harmattan, Paris. 


\section{Le rôle de l'activité de régulation sociale dans le processus d'affirmation de nouvelles parties prenantes: l'exemple des réseaux de promotion de la diversité}

Berthoin Antal A. \& Sobczak A. (2004), « Beyond CSR: Organizational learning for global responsibility », Journal of General Management, Vol. 30, No 2, 77-98.

Brass D. J. (1985). « Men's and Women's Networks: a Study of Interaction Patterns and Influence in Organization ", Academy of Management Journal, Vol. 28, N² 2, 327-343.

Brechet, J.-P. (2008). " Le regard de la théorie de la régulation sociale de Jean-Daniel Reynaud ", Revue Française de Gestion, No 184, 13-34.

Chanlat J.F. (2005). " La recherche en gestion et les méthodes ethnosociologiques ", in Management des Ressources Humaines. Méthodes de recherche en sciences humaines et sociales. De Boeck, 159-175

Chollet B. (2006). « Qu'est-ce qu'un bon réseau personnel ? Le cas de l'ingénieur R\&D », Revue Française de Gestion, No 163, 107-125.

Commission Européenne. (2002), Communication sur la responsabilité sociale des entreprises: Une contribution des entreprises au développement durable, COM/2002/0347 final, Bruxelles.

Cornet A. et Warland P. (2008). GRH et gestion de la diversité, Dunod, Paris.

Cox T. (1993). Cultural Diversity in Organizations: Theory, Research and Practice, BerrettKoehler Publishers, San Francisco.

Delamont S. (2004). " Ethnography and Participant Observation », in C. Seale and al. (eds.), Qualitative Research Practice, Sage Publication, 217-229.

Freeman R. E. (1984), Strategic Management: A Stakeholder Approach, Pitman, Boston. Girard, C. et Sobczak, A. (2010). « Pour une cartographie des parties prenantes fondée sur leur engagement : Une application aux sociétaires d'une banque mutualiste française ", Management \& Avenir (à paraître dans ce même numéro)

Granovetter M. (1983). " The strength of weak ties: a network theory revisited ", Sociological Theory, Vol. 1, 201-233.

Groleau C. (2003). « L'observation », in Y. Giordano (dir.), Conduire un projet de recherche. Une perspective qualitative, EMS. Éd. Management \& Société, Colombelles, 213-244.

Havard C. et Krohmer C. (2008). "Création et articulation des règles dans le cadre d'un management des compétences », Revue de Gestion des Ressources Humaines, No 70, 88-101.

Ibarra H. (1993). «Personal networks of women and minorities in management: a conceptual framework », Academy of Management Review, Vol. 18 No 1, 56-87.

IMS-Entreprendre pour la Cité. (2006), « Gestion de la diversité - Le cercle de la diversité», Communication aux Deuxièmes Rencontres Internationales de la Diversité, 5-7 octobre 2006.

Landrieux-Kartochian S. (2007), « Quel business case pour la féminisation des entreprises ? ", in I. Barth et C. Falcoz, Le management de la diversité, enjeux, fondements et pratiques, L'Harmattan, Paris.

Laufer J. (2005). « La construction du plafond de verre : le cas des femmes cadres à potentiel », Travail et Emploi, No 102, 30-44.

Marchal, E. et Rieucau, G. (2009). « Formes d'intermédiation et formes de sélection : les contrastes entre annonces et réseaux de relations », Economies et Sociétés, Série "Socio-Economie du Travail », AB, No 30, 3-26.

Mitchell R. K., Agle B. R. \& Wood D. J. (1997). " Toward a Theory of Stakeholder Identification and Salience: Defining the Principle of Who and What Really Counts", Academy of Management Review, Vol. 22, No 4, 853-886.

Naschberger C. (2008). "La mise en œuvre d'une démarche "diversité en entreprise » - Le cas de l'intégration des personnes en situation de handicap » Management \& Avenir, No $18,42-56$.

Peretti J.-M. et Cerdin, J. (2005). Performances économiques et performance sociales à l'heure de la R.S.E. Cergy-Pontoise, Essec Business School.

Reynaud J.-D. (1979). "Conflit et régulation sociale. Esquisse d'une théorie de la 
régulation conjointe », Revue Française de Sociologie, Vol. 20, 367-376.

Reynaud J.-D. (1988). « Les régulations dans les organisations : régulation de contrôle et régulation autonome ", Revue Française de Sociologie, Vol. 29, 5-18.

Reynaud J.-D. (1991). "Pour une sociologie de la régulation sociale », Sociologie et Sociétés, Vol. 23, No 2, 13-26.

Reynaud J.D. (1997). Les règles du jeu. L'action collective et la régulation sociale, $2^{\text {ème }}$ édition, Armand Colin, Paris.

Reynaud J.-D. (1999). Le conflit, la négociation et la règle, Octarès Éditions, 2ème édition, Paris.

Reynaud J.-D. (2003). « Régulation de contrôle, régulation autonome, régulation conjointe ", in G. de Terssac (dir.), La théorie de la régulation sociale de Jean-Daniel Reynaud, Paris : La Découverte, 103-113.

Reynaud J.-D. (2005). "Ce que produit une négociation collective, ce sont des règles ", Revue Négociations, No 2, 141-159.

Reynaud J.-D. \& Richebé, N. (2007). « Règles, conventions et valeurs. Plaidoyer pour la normativité ordinaire ", Revue Française de Sociologie, Vol. 48, No 1, 3-36.

Schoemann I., Sobczak A., Voss E., Wilke P. (2008). Codes of conduct and international framework agreements: New forms of governance at company level, Report to the European Foundation for the Improvement of Living and Working Conditions.

Seidel M. D. L., Polzer J. T. \& Steward K. J. (2000). « Friends in the High Places: the Effects of Social Networks on Discrimination in Salary Negotiations », Administrative Science Quarterly, Vol. 45, 1-24.

Sobczak A. (2002). Réseaux de sociétés et codes de conduite : un nouveau modèle de régulation des relations de travail pour les entreprises européennes, LGDJ, coll. Bibliothèque de Droit social, tome 38 , Paris.

Sobczak A. (2010). Multiplier les perspectives pour enrichir l'analyse des pratiques de responsabilité sociale des entreprises, Habilitation à Diriger des Recherches, Université Paris 12. 
Copyright of Revue Management et Avenir is the property of Management Prospective Editions and its content may not be copied or emailed to multiple sites or posted to a listserv without the copyright holder's express written permission. However, users may print, download, or email articles for individual use. 